\title{
Thanks to geolocalized remote control : the sound will follow
}

\author{
Mathieu Hopmann \\ mathieu.hopmann@epfl.ch
}

\author{
Daniel Thalmann \\ daniel.thalmann@epfl.ch \\ VRLab - EPFL \\ 1015 Lausanne \\ Switzerland
}

\author{
Frederic Vexo \\ frederic.vexo@epfl.ch
}

\begin{abstract}
Dematerialized music and MP3 players offer to the user the possibility of carrying his musical content everywhere. However, at home, the music collection is generally not centralized, split between different rooms and audio supports (CDs, MP3s, etc). In this paper, we propose a multi-room sound system based on several sound satellites distributed among the rooms of the house, and on a remote control which is able to localize itself with the help of available Wi-Fi networks. The user only has to choose his favorite music on the remote control, and then the music will automatically start on the nearest satellites. Our goal is to offer an Ambient Intelligence audio system, inspired by the latest multi-room sound systems, which takes advantage of an indoor localization system based on Wi-Fi in order to propose a new experience to the user.
\end{abstract}

\section{Introduction}

Music has become more and more dematerialized over last years due to the development of $\mathrm{P} 2 \mathrm{P}$ file sharing and MP3 players. Thanks to these devices, the music follows the user wherever he is : he could access to his entire musical content in the bus, in the street, or in his car by plugging his player on the head unit.

At home, sound systems in different rooms are often completely independent. Each system needs its own remote control, and each system could only play the music on the inserted $\mathrm{CD}$ or on the hard drive. The music collection is often split between CDs, PCs and MP3 players in different rooms.

With the development of Internet, Wi-Fi networks at home become widely available. If the user can access to streaming music over the Internet, why can't he access to his entire music collection everywhere in his house?
In this paper we propose a multi-room sound system which takes advantage of indoor remote control localization in order to provide smart sound follow experience. The location of the user is delivered by the analysis of Wi-Fi signal strength values and a semantic graph describing the environment topology. The music collection is centralized on one device, the main sound satellite, and is completely accessible from everywhere in the house thanks to the remote control.

The paper is organized as follows : first we present a short description of ambient intelligence, to show the benefits of this concept on everyday's devices like music players. We continue with a complete description of our application : presentation, hardware description and technical approach. Last section concerns our experiments and the validation of our proof of concept.

\section{Related works}

At the end of the 90s emerged the concept of Ambient Intelligence[1][8]. This concept, firstly developed by Philips, involves the presence of surrounding electronic devices in everyday life which react with the user behavior and habits in the most natural way. To refine the notion of Ambient Intelligence, Aarts and Marzano[2][3] defines the key technology features as : Embedded, Context aware, Personalized, Adaptive and Anticipatory.

Recently, one of the first products following the Ambient Intelligence paradigm has been widely available. They are multi-room music systems, such as the Sony S-AIR system, the Philips Streamium wireless music center $^{1}$ or the Logitech SqueezeBox. These systems are often divided in several components : the main unit with the music collection, and smaller unit connected wirelessly to the previous one and able to play all the music contained on it by using the UPnP (Universal

\footnotetext{
${ }^{1}$ http://www.streamium.com/
} 
Plug and Play) network protocol. This way, the user could have access to his entire musical content wherever he is.

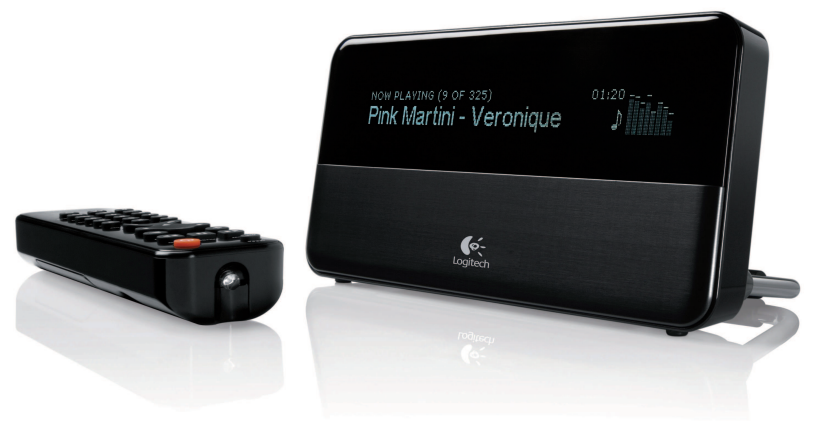

Figure 1. An example of multi-room sound system : the Logitech Squeezebox

These multi-room music systems match different Ambient Intelligence key features. The music is embedded in the environment, not on a physical format such as CDs or DVDs, allowing the user to access all of his music collection everywhere and without constraints. The personalized and adaptive features are given by the remote control : the user chooses his music and the remote control will play the music on the pointed device.

Speaking about Ambient Intelligence audio systems, we could take the example of the Philip's Streamium device. One of the Streamium advanced mode is the "Music follows me" system. To achieve that, the user pushes the "Music follows me" button one time in the current room, and one time in his destination room, and the music is transferred from the first room to the second room. By this way, this system partially benefits of the context awareness, but requires a user interaction to be possible. And despite the "Music follows me" slogan, their concept is based on the music transfer and not on music that completely follows the user. This interaction process could strongly take advantage of an invisible indoor geolocation system, allowing a fully automated process and a real follow of the user. It refers to the context aware[4] and anticipatory features : the music follows the user with the location system, and the system anticipates user desires without conscious mediation by modifying dynamically the music volume. That's why we will now study indoor location system alternative.

Classically, the most commonly used geolocation system is the Global Positioning System (GPS). However, this system is designed to be functional only outdoor, whereas Ambient Intelligence is mostly oriented in indoor use. Indoor localization and tracking of persons begin many years ago with specialized hardware requirements (infrared, ultrasound, electromagnetism, etc), which are precise but also really expensive and often limited to one room or less. Recent systems tried to avoid these cost and deployment problems by using technologies already deployed such as GSM, Wi-Fi or Bluetooth. One of the first works in this direction was RADAR [6] [7], a tracking system based on signal strength information given by radio frequency devices. RADAR system worked in two phases: the off-line phase which consisted in collecting signal strength data in order to build a signal map, and the real-time phase, corresponding to the localization phase and based on the previously acquired signal map. This system provided a median resolution of nearly 3 meters. This twophase model has inspired several papers, which tried to enhance this system in different manners and for different purposes: enhance accuracy and precision [5] [10], localize in high signal fluctuation areas [9], use only one access point [11]. Most of these papers are based on the Wi-Fi technology, and shows that localization using this technology is conceivable. Our idea is to implement our Wi-Fi localization system, and use it to provide context awareness to applications which could take advantage of this information.

Next section will describe our choices and implementation in order to integrate location awareness into multi-room music systems.

\section{Sound will "really" follow}

In this section we first present the fundamental concept supporting our prototype, and then describe the technical aspect of the application.

\subsection{Description of the concept}

Our multi-room sound system is based on several sound satellites distributed among the rooms of the house and a remote control which is able to localize itself with the help of available wireless networks.

Our application scenario : the user selects threw the remote control a music to be played or a playlist. Then, when he is moving in the house, he carries the remote with him and the remote localizes itself and transmits the position to the satellite to start or mute them. A satellite which is too far away from the user is mute. When the user is in a room with a satellite, the satellite starts playing. Finally, when the user is in a room or a corridor without a satellite, closest satellites plays music with a volume which depends on the distance between the user and the satellites. 


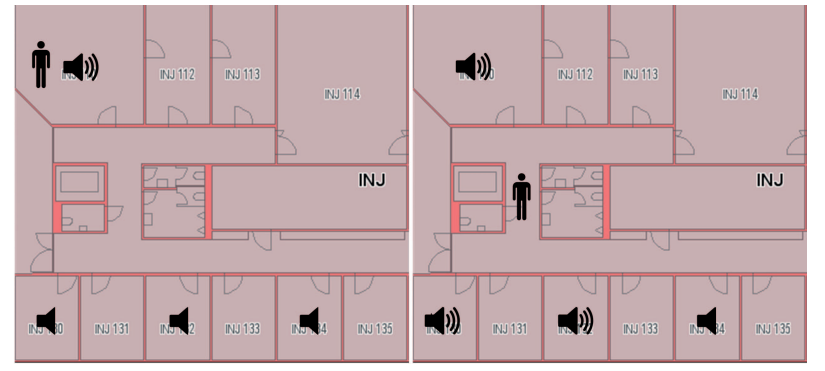

Figure 2. The figure shows the influence of the user position on the sound system activation

\subsection{Implementation}

\subsubsection{Hardware description}

To perform our experiments, we needed two kind of hardware responding to these points :

Remote control The remote control must be pocket size, with a screen big enough to display clearly the user interface. It must have Wi-Fi capabilities to communicate with the sound devices and to be localized.

Sound player The sound players must be connected to the network in order to communicate with the remote control.

As proof of concept, we chose the Ultra-Mobile PC (UMPC) Sony VAIO UX90 for the remote control. This device offers more hardware and software freedom compared to other handheld devices such as smart phones or PDAs : more power (CPU, memory), USB connectivity, choice of operating system, choice of development tools. The main drawback is its size, which is not really adapted for the pocket, but this is not an issue for our tests.

The Wi-Fi chipset embedded on the Ultra Mobile $\mathrm{PC}$ was not satisfying to perform localization: the signal strength values range was short (between -50 and $-90 \mathrm{~dB}$ ) compared to the chipset embedded on an Intel Centrino laptop for example (between -30 and $-90 \mathrm{db}$ ). For this reason, we looked for a Wi-Fi USB adapter, and chose after some tests the Linksys WUSB200, which provides a better value range (equivalent to the Centrino). Another advantage of having a second WiFi chipset is the network connection possibility. Indeed, when you are associated with an access point, the values are updated less quickly or only for the linked access point.

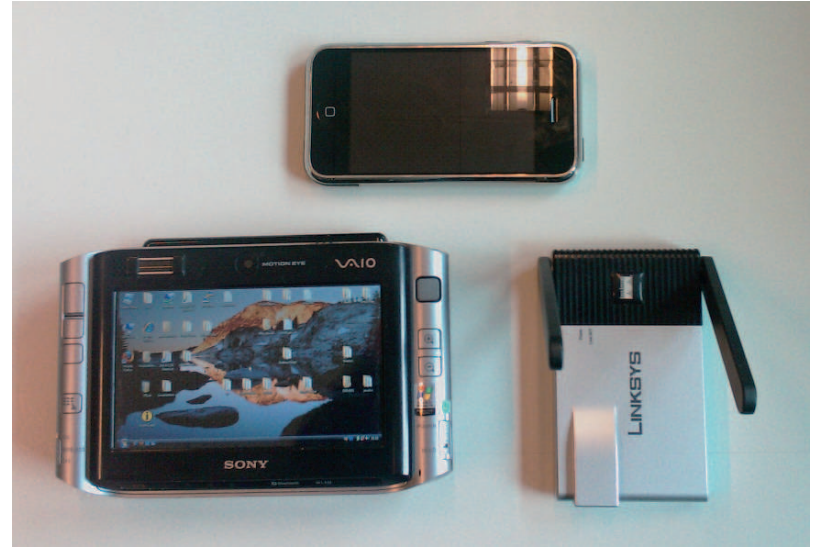

\section{Figure 3. UMPC Sony VAIO UX90 and Linksys} WUSB200. The scale is given by the iphone

Finally, our sound players are PCs connected on our network. The advantage is the ability to easily communicate with the remote. Our application doesn't do complex computation on these PCs, the tasks are limited to play, mute, or change the volume of the music.

\subsubsection{Sofware implementation}

User positioning The first software we have to integrate in the remote control is the localization algorithm. To begin, we need to collect signal strength values. In order to do this, we choose Vista : Microsoft provides with its operating system a new API, the Native Wi-Fi $\mathrm{API}^{2}$. This interface offers functions to update and access Wi-Fi information, which is precisely what we are looking for. The only drawback is a slower Wi-Fi refresh rate when the adapter is connected to an access point. This point is not really annoying for us because of our two Wi-Fi chipsets : the WUSB200 to read WiFi information, and the UMPC chipset for the network connection. Concretely, with the Windows Native Wi-Fi API and our Linksys WUSB200, the Wi-Fi information (BSSID and RSSI) are updated one time per second.

About our localization software, our needs are a program which is :

Light Our program is intended to run on a handheld device

Fast The music has to follow the user, so we need to update user position as fast as possible.

\footnotetext{
${ }^{2}$ http://msdn2.microsoft.com/enus/library/ms706556(VS.85).aspx
} 
Accurate Precision is not our priority, but we want a reliable position to always play music in the user current room.

As other radio frequency positioning systems, our system works in two phases: the off-line phase which consists in collecting signal strength data in order to build a signal map, and the real-time phase, corresponding to the localization phase and based on the previously acquired signal map. During the off-line phase, the user creates a navigation graph: each room (or part of corridor) is represented as a vertex of the graph, then the user has to link neighbour vertices between them and records signal data (access point identifiers and signal strengths) for each vertex.

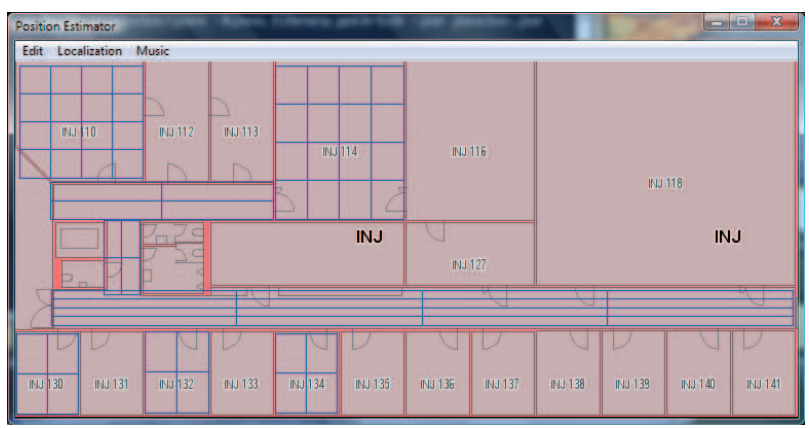

Figure 4. The editor allows the calibration of Wi-Fi information and sound players position

After this quick training phase (15 seconds per vertex to capture signal data), the localization system is ready to use: the application takes $\mathrm{Wi}-\mathrm{Fi}$ samples every second during three seconds, smoothes the received signal strengths by removing the values too far from the mean if the standard deviation is too high, locates the user (by a simple distance calculation) according to the signal map acquired during the off-line phase. The system uses the navigation graph to ensure that the new position is realistic (close to the previous one), and then avoid the biggest mistakes due to signal noise.

We will now describe the communication between the remote and sound players.

The first communication is the interaction between the remote control and the main sound player, which contains the music file, in order to select the current music to play, and diffused it on the network. The second interaction is between the remote control and all the sound players : the remote control decides which player is on and the volume of the current players. Figure 5 shows the interaction between each device.

To facilitate the communication between all the de- vices, we choose Java RMI (for Remote Method Invocation $^{3}$ ) : this programming interface allows applications to easily access distant objects. In our case, it is helpful to dynamically turn on/off a music player or change its current volume from the remote control.

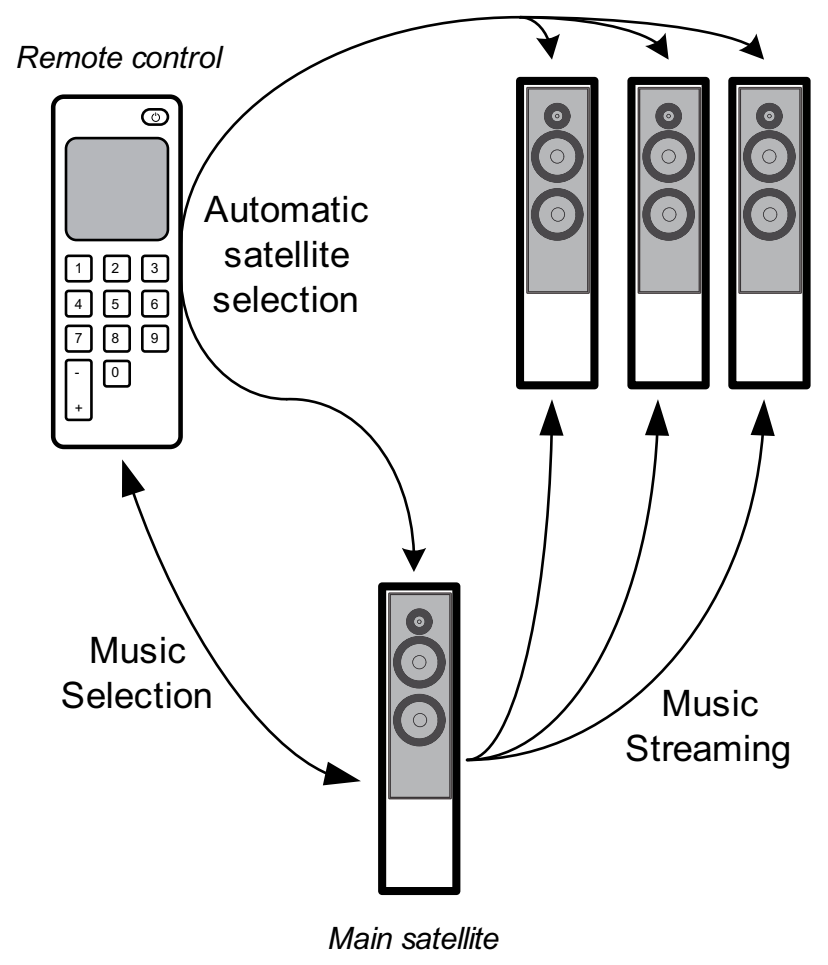

Figure 5. Illustration of our architecture

Music diffusion For the music diffusion, we have one RMI client and one RMI server.

The RMI server is the main music player, which contains all the music files, and offers three services : a function to list the music files, a function to start the music diffusion and a function to stop the music diffusion. When the server has to start the music diffusion, the selected file is diffused on the network using the multicast RTP protocol : this way, all the devices connected on the same network are able to play the music diffused by the main music player. To diffuse the music using multicast RTP protocol, we choose Java Media Framework $\left(\mathrm{JMF}^{4}\right)$, which is not supported anymore but still efficient.

The RMI client is the remote control : when the user wants to access the music database, the remote control asks the server to list all the files. Then, the user chooses his music, and could start the music dif-

\footnotetext{
${ }^{3}$ http://java.sun.com/javase/technologies/core/basic/rmi/index.jsp

${ }^{4}$ http://java.sun.com/products/java-media/jmf/index.jsp
} 
fusion. At this moment, the main music player begins to start the diffusion over the network, and the remote control starts to localize the user in order to activate adapted players.

Sound players activation For the sound player activation, we have one RMI client for the remote control and one RMI server for each music player.

Each server allows the client only to start or stop the music. The start function takes as input the volume given by the client. When the client asks to the server to start the music, the server just read the multicast RTP flow (sending by the main satellite) using JMF.

The client, based on the remote control, reacts each time a new position is evaluated (i.e. every three seconds as we say in subsection 3.2.2). If the new position is in a room containing a music player, the client turns only this music player on. On the contrary, if the user is in a room without a music player (it could be a corridor for example), each close player (for our experiments, we choose a distance of less than 20 meters) is turned on, with an increased volume. To calculate the new volume, we are using the sound attenuation formula : this formula is normally reserved to environment without obstacles, but it gives a good idea on how we have to increase the volume to compensate the distance. The formula is

$$
V 2=V 1-20 \log \frac{D 2}{D 1}
$$

with V1 (dB) and D1 the original volume and distance, and V2 (dB) and D2 the new volume and distance. When the user modifies the volume of the music, he/she modifies the V1 parameter.

\subsection{Experiments and validation}

The system was tested in our laboratory. The surface is 817 square meters $(43 \mathrm{~m} 19 \mathrm{~m})$, representing 18 offices of variable size. The floor is directly covered by 4 access points ( 2 Cisco Aironet 1200 and 2 NETGEAR WGR614), but we can acquire up to 7 different signals in some rooms (from access points placed on the nearest floors or buildings).

To test our system and have a feedback on it, we asked 12 persons to use our music application. The purpose of the experiment was firstly to test the accuracy of our localization system, and secondly to see how the system was immersive.

Users were asked to check the position shown in the UMPC and qualifying the systems accuracy as wrong, near or good. The good qualification was applied if the localization system shown the exact room or corridor
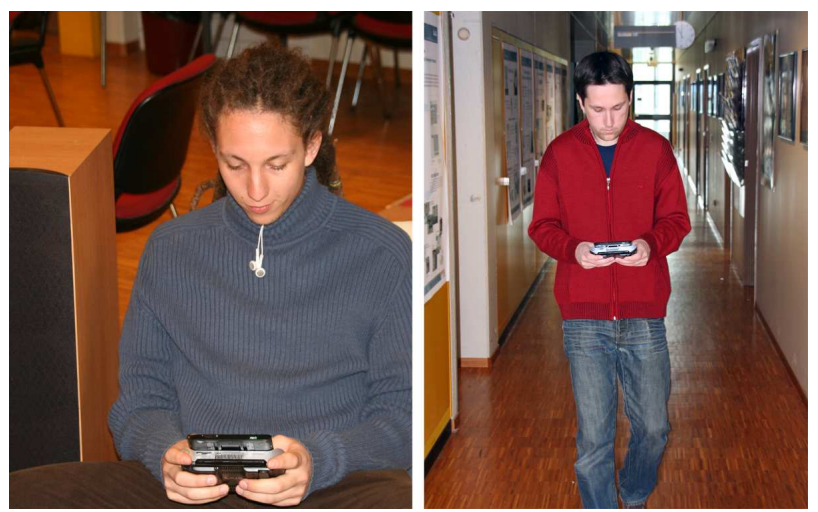

Figure 6. Users interacting with our multiroom sound system

part, the near qualification when the system indicated not the exact position but a juxtaposed position, and the wrong was for all other cases.

Table 1. Localization experiment result

\begin{tabular}{|c|c|c|}
\hline Good & Near & Wrong \\
\hline \hline $81 \%$ & $15 \%$ & $4 \%$ \\
\hline
\end{tabular}

Table 1 shows the result of our localization experiment. During these tests, we have noticed that the $15 \%$ near and $4 \%$ wrong were mostly obtained in the corridor, because of the lack of important changes in the signal compared to pass a door for example. Mistakes in corridor are not really annoying because they only influence the sound volume of the music players. But mistakes in rooms are a real issue : even if it happens rarely, it is really upsetting for the user to have the music playing in the wrong room. Unfortunately, these mistakes can't be completely removed with a localization system only based on $\mathrm{Wi}-\mathrm{Fi}$, because noise will always occurs. For this reason, we think that add a second localization system could be helpful : a short-range localization system (based on RFID tags for example) to ensure user position in room with music players, and Wi-Fi for corridor or rooms without sound players.

\section{Conclusion}

In this paper, we have developed a multi-room music system which takes advantage of an indoor localization system based on Wi-Fi to allow sound follow. Our application, which follows the main Ambient Intelligence concepts, uses existing $\mathrm{Wi}-\mathrm{Fi}$ access points in order to localize the user's remote control in the house. The user position allows the program to select on which 
sound systems the music will be played, and to modify the sound volume if necessary. Our experiment proves the concept of using indoor localization to enhance the user experience.

In a future work, we could easily switch from a speaker attached to a PC to an existing audio satellite system working on UPnP. By the same way, we could easily replace the UMPC by a device closer to a universal remote control size, like the Logitech Harmony One.

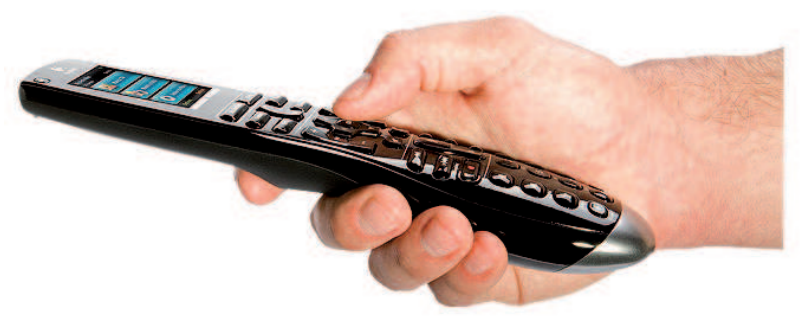

Figure 7. Logitech Harmony One

Our localization system works well in an urban environment where we are able to acquire numerous $\mathrm{Wi}-\mathrm{Fi}$ networks, but the precision and accuracy drop down dramatically in an individual house where only one access point is visible at a time. It's the reason why we will work in the future on a short-range RF module which could be inserted in the satellites and in the remote in order to give proximity information to the localization system.

\section{Acknowledgement}

This work has been supported by the Swiss National Science Foundation (FNS) under the framework of "Semantic Based Tangible interface: "the remote control paradigm"" project.

\section{References}

[1] E. Aarts, R. Harwig, and M. Schuurmans. Ambient intelligence. pages 235-250, 2002.

[2] E. Aarts and S. Marzano. The New Everyday: Views on Ambient Intelligence. 2003.

[3] E. Aarts, P. Res, and N. Eindhoven. Ambient intelligence: a multimedia perspective. Multimedia, IEEE, 11(1):12-19, 2004.
[4] G. Abowd and A. Dey. Towards a Better Understanding of Context and Context-Awareness. Handheld and Ubiquitous Computing: First International Symposium, HUC'99, Karlsruhe, Germany, September 1999: Proceedings, 1999.

[5] J. Astrain, J. Villadangos, J. Garitagoitia, J. de Mendívil, and V. Cholvi. Fuzzy location and tracking on wireless networks. Proceedings of the international workshop on Mobility management and wireless access, pages 84-91, 2006.

[6] P. Bahl and V. Padmanabhan. RADAR: an inbuilding RF-based user location and tracking system. INFOCOM 2000. Nineteenth Annual Joint Conference of the IEEE Computer and Communications Societies. Proceedings. IEEE, 2, 2000.

[7] P. Bahl, V. Padmanabhan, and A. Balachandran. Enhancements to the RADAR User Location and Tracking System. Microsoft Research, 2000.

[8] K. Ducatel, M. BOGDANOWICZ, F. SCAPOLO, J. LEIJTEN, and J. BURGELMAN. ISTAG Scenarios for Ambient Intelligence in 2010. Final Report. European Commission, Feb, 2001.

[9] W. Ho, A. Smailagic, D. P. Siewiorek, and C. Faloutsos. An adaptive two-phase approach to wifi location sensing. In PERCOMW '06: Proceedings of the 4 th annual IEEE international conference on Pervasive Computing and Communications Workshops, page 452, Washington, DC, USA, 2006. IEEE Computer Society.

[10] R. Kumar K., V. Apte, and Y. Powar. Improving the accuracy of wireless lan based location determination systems using kalman filter and multiple observers. Wireless Communications and Networking Conference, 2006. WCNC 2006. IEEE, 1:463-468, 0-0 0 .

[11] G. V. Zàruba, M. Huber, F. A. Kamangar, and I. Chlamtac. Indoor location tracking using rssi readings from a single wi-fi access point. Wirel. Netw., 13(2):221-235, 2007. 